\title{
Pensamiento reflexivo del estudiante de enfermería en su prácticum clínico'
}

\author{
Luz Nelly Rivera Álvarez² \\ José Luis Medina Moya
}

doi:10.11144/Javeriana.ie19-1.pree

Cómo citar: Rivera Álvarez LN, Medina Moya JL. Pensamiento reflexivo del estudiante de enfermería en su prácticum clínico. Investig Enferm. Imagen Desarr. 2017; 19 (1): 1730. http://dx.doi.org/10.11144/Javeriana.ie19-1.pree

1. Articulo original de investigación. Fecha de recepción: 21 de Agosto de 2015 Fecha de aprobación: 27 de Enero de 2016.

2. Profesora asociada del Departamento de Enfermeria, Facultad de Enfermeria, Universidad Nacional de Colombia. Grupo de investigación Salud y Cuidado de los Colectivos. Dirección: Cra. 30 \# 45-03, Edificio 228, Bogotá, Colombia. Correo electrónico: lnriveraa@unal.edu.co.

3. Profesor titular del departamento de Didáctica y Organización Educativa, Facultad de pedagogía, universidad de Barcelona. Correo electrónico: jlmedina@ub.edu 


\section{Resumen}

Objetivos: Describir cómo emergen los procesos de pensamiento reflexivo del estudiante de enfermería ante situaciones clínicas en su prácticum clínico. Método: Investigación cualitativa, hermenéutico-fenomenológica, realizada en la asignatura Practicum Hospitalario II durante las prácticas clínicas de once estudiantes de enfermería de una Facultad de Enfermería y un Hospital Universitario de Barcelona (España), de febrero de 2011 a febrero de 2012. Las estrategias de recogida de datos fueron: observación participante, observación no participante, conversación informal, entrevista en profundidad y materiales escritos. Para el análisis de la información se empleó el método de comparaciones constantes de Strauss y Corbin. Resultados: El eje cualitativo emergente fue: Convergencia de significados entre estudiante y enfermera respecto al cuidado competente en enfermería; y la metacategoría resultante fue: Pensamiento reflexivo del estudiante, con las subsiguientes categorias: capacidad perceptiva y consciencia situada de las necesidades del otro; curiosidad epistemológica; atención operativa; la anticipación: conversación reflexiva en la acción; y finalmente imitación reflexiva. Conclusiones: La observación deliberativa, la atención operativa, la conversación reflexiva en y sobre la acción, la curiosidad epistemológica y la imitación reflexiva, emergen de manera gradual y acorde a la calidad en la continuidad e interacción del estudiante con los diferentes elementos de su prácticum clínico; estos procesos reflexivos propician en el estudiante la posibilidad de reflexionar, interpretar y significar su propia práctica.

Palabras clave: investigación en educación de enfermería; enseñanza. Aprendizaje; pensamiento; investigación cualitativa

\section{Reflective thinking of the nursing student in the clinical practicum}

\section{Abstract}

Objectives: To describe how the processes of reflective thinking emerge in the nurse student in face of clinical situations in their clinical practicum. Method: Qualitative research, hermeneutical-phenomenological made in the subject Hospital Practicum II during the clinical practicum to eleven nursing students at a Faculty of Nursing and a University Hospital of Barcelona (Spain), from February 2011 to February 2012.The data collection strategies were: participant observation, non-participant observation, informal discussion, in-depth interviews and written materials. For the data analysis, the method of constant comparisons by Strauss and Corbin was applied. Results: The emerging qualitative axis was: Convergence of meanings between student and nurse regarding competent nursing care; and the resulting metacategory was: thoughtful student thinking with subsequent categories: perceptiveness and consciousness regarding the needs of others; epistemological curiosity; operative care; anticipation: reflective conversation in action; and finally reflexive imitation. Conclusions: Deliberative observation, operational attention, reflective conversation about action and in it, epistemological curiosity and reflective imitation, emerge gradually and in accordance to the quality of continuity and the student interaction with the different elements of their clinical practicum; these reflective processes foster in the student the opportunity to reflect, interpret and give meaning to their own practice.

Keywords: nursing education research; teaching. Learning; Thought; qualitative research 


\section{Pensamento refletivo do estudante de enfermagem no seu prácticum clínico}

\section{Resumo}

Objetivos: Descrever a forma como os processos de pensamento reflexivo do estudante de enfermagem emergem em face de situações clínicas no seu prácticum clínico. Método: Pesquisa qualitativa, hermenêutico-fenomenológica, realizada na matéria Prácticum Hospitalar II durante as práticas clínicas de onze alunos de enfermagem de uma Faculdade de Enfermagem e um Hospital Universitário de Barcelona (Espanha), de fevereiro de 2011 a fevereiro de 2012. As estratégias para a coleta de dados foram: observação participante, observação não participante, conversa informal, entrevista em profundidade e materiais escritos. Para a análise da informação utilizou-se o método de comparações constantes de Strauss e Corbin. Resultados: O eixo qualitativo emergente foi: Convergência de significados entre aluno e enfermeiro no que diz respeito ao cuidado competente em enfermagem; e a metacategoria resultante foi: Pensamento refletivo do aluno com as subsequentes categorias: capacidade perceptiva e consciência situada das necessidades do outro; curiosidade epistemológica; atenção operativa; a antecipação: conversa reflexiva na ação; e por fim, imitação refletiva. Conclusões: A observação deliberativa, a atenção operativa, a conversa refletiva em e sobre a ação, a curiosidade epistemológica e a imitação refletiva, emergem de maneira gradual e de acordo com a qualidade na continuidade e interação dos alunos com os diferentes elementos do seu prácticum clínico; estes processos refletivos propiciam no aluno a possibilidade de refletir, interpretar e significar sua própria prática.

Palavras-chave: pesquisa em ensino de enfermagem; ensino. Aprendizagem; pensamento; pesquisa qualitativa 


\section{Introducción}

La formación clínica del estudiante de enfermería necesita trascender la idea de instrucción o de enseñanza para la fabricación; es decir, superar la racionalidad técnica. Entendiendo que la formación hace alusión a un proceso interno y no a los resultados, se vuelca hacia la experiencia educativa entre el educando y el educador, y problematiza el significado de cuidar a otro, a diferencia de la instrucción, que se preocupa por el aprendizaje meramente teórico y técnico.

La formación corresponde, entonces, a un obrar y no-obrar (en una deliberación ética, estética e histórica del sujeto) que se ajusta a lo esencial y relevante en cada situación educativa. Esta deliberación permite la formación de hábitos reflexivos, atentos y precisos de pensamiento, amplia la preferencia por las conclusiones que son adecuadamente fundamentadas y por un hacer sistemático, razonado y apropiado a las diversas situaciones que se presentan (1).

Las actitudes necesarias para provocar un pensamiento reflexivo, son: "apertura de mente", que exige el "principio de escucha activa"; "entusiasmo", como categoría básica de la motivación intrínseca; y "responsabilidad intelectual", como la capacidad para responder a las consecuencias de una determinada proposición (1). La conjunción de estas tres actitudes éticas, además de permitir la formación de una estructura cognitiva de moralidad, facilita la aparición de un pensamiento genuinamente reflexivo. Tal estructura exige la apropiación de un pensamiento pedagógico práctico-prudencial orientado a: la correcta regulación ética de los procesos de deliberación, de formulación de juicios educativos y de toma de decisiones pedagógicas; la maduración; la conformación ética y psíquica de la personalidad como educadores (2). Algunos autores (1-4) apuntan a que el pensamiento reflexivo comienza en una situación de incertidumbre o situación problemática, caracterizada ésta por la perplejidad, en la que justamente se origina la observación e indagación deliberativa y el análisis crítico de la situación, para así localizar la naturaleza del problema; seguidamente se plantean las hipótesis, los objetivos concretos, la elaboración de algún juicio final y las alternativas de solución para esclarecer el problema; y, por último, la corroboración experimental, o experimentación in situ (3). Teniendo en cuenta lo anterior y a la búsqueda bibliográfica, se encuentra que existen pocas investigaciones sobre el proceso reflexivo del estudiante de enfermería; el rol del contexto en el proceso reflexivo; los aspectos más significativos de la enseñanzaaprendizaje del estudiante al interactuar con los pacientes; los resultados de la práctica reflexiva en el cuidado del paciente (5-8); y cómo el cuidado es percibido, aprendido y practicado por los estudiantes en dichas interacciones $(9,10,11)$. Solo algunos estudios han abordado la práctica reflexiva del estudiante de enfermería, los cuales muestran que estimular a los estudiantes a reflexionar sobre su propia práctica favorece a: expresar sus sentimientos y necesidades de aprendizaje, compartir experiencias, solucionar problemas e identificar razones para la acción $(5,7)$. Por tanto, nos preguntudiante de enfermería en su práctica clínica? y planteamos como objetivo 
de la investigación: describir cómo emerge el pensamiento reflexivo del estudiante de enfermería ante las situaciones clínicas -en interacción con el paciente- en su prácticum.

\section{Método}

Investigación cualitativa, con abordaje teórico fenomenológico- hermenéutico (12) y aproximación metodológica etnográfica. Para la selección de las participantes del estudio empleamos la selección simple basada en criterios. En el estudio participaron una profesora supervisora, tutoras clínicas y once estudiantes de tercer año que cursaban la asignatura $l$, en una Facultad de Enfermería de Barcelona (España), quienes realizaban sus prácticas clínicas en un hospital universitario. En este contexto la profesora supervisora tiene a su cargo la supervisión clínica de un grupo de seis a ocho (6 a 8) estudiantes y la tutora clínica (enfermera) es quien tiene bajo su responsabilidad la formación práctica de un estudiante. Las estrategias de recogida de la información fueron la observación participante y no participante, la conversación informal, la entrevista en profundidad y el análisis de documentos (diarios reflexivos de los estudiantes, plan curricular de la asignatura). La recogida de datos empleó el criterio de la saturación teórica y se desarrolló en dos grupos de estudiantes y en dos fases. La primera fase estuvo comprendida entre febrero de 2011 y julio de 2011, con un grupo de cinco estudiantes (Grupo I); y la segunda fase desde septiembre de 2011 hasta febrero de 2012, con un grupo de seis estudiantes (Grupo II). Se realizaron 44 horas de observación no participante durante los seminarios de la práctica, esta observación fue realizada en un aula de la facultad de enfermería, donde se observó la acción pedagógica entre el grupo de estudiantes y la profesora al analizar las situaciones clínicas vivenciadas; esta observación se realizó en las dos fases y en los dos grupos de estudiantes. En la primera fase se realizaron 20 horas de observación y en la segunda 24 horas de observación no participante; adicionalmente, se realizaron 95 horas de observación participante durante las prácticas clínicas de las estudiantes y conversaciones informales antes, durante y posterior a dicha observación; y por último, se realizaron entrevistas semi-estructuradas en profundidad a cada estudiante (excepto a una estudiante, a quien fue necesario realizar dos entrevistas), una entrevista a una enfermera tutora y una entrevista a la profesora supervisora, consideradas estas como participantes claves, para un total de 14 entrevistas. El análisis de la información siguió los tres momentos del método de comparaciones constantes (13). En el primero, se realiza la codificación abierta, para identificar categorias; en el segundo se realiza la codificación axial, donde se interrelacionan las categorías para identificar los núcleos temáticos emergentes; y por último, la codificación selectiva, donde se identifican los ejes cualitativos centrales que atraviesan el corpus de datos. Las observaciones y entrevistas fueron transcritas y organizadas en el programa Atlas ti versión 6. Las estrategias de rigor metodológico que se siguieron para asegurar la credibilidad o validez interna del estudio fueron: estancia prolongada en el campo clínico y observación persistente; por medio de la triangulación (de métodos, de participantes e investigadora, y con 
expertos) y la corroboración estructural y adecuación referencial de los resultados. El estudio fue revisado por el Comité de Ética en Investigación del Hospital Universitario -aprobado el 9 de junio de 2011 (Acta 11/11) con el $n^{\circ}$ PR144/11-; siguió los principios éticos contemplados en la Declaración de Helsinki de la Asociación Médica Mundial (14) y la Ley orgánica española de Protección de Datos de Carácter Personal (LOPD) en su Título III (15). Los participantes aceptaron participar a través del consentimiento informado por escrito. Los nombres de los participantes fueron sustituidos por nombres ficticios.

\section{Resultados y Discusión}

La convergencia de "significados" entre estudiante y enfermera tutora respecto al cuidar competente en enfermería, emergió como el eje cualitativo que atravesaba los datos. Dicha formación competente tiene en cuenta el pensamiento reflexivo del estudiante, el cual emergió como metacategoria, y hace referencia a aquellos procesos reflexivos del estudiante que emergen en el interior de su práctica de cuidado. El pensamiento reflexivo del estudiante se manifestó con las categorias que se describen en los siguientes apartados.

\section{Capacidad perceptiva y consciencia situada de las necesidades del otro}

A continuación se presenta una situación de incertidumbre, en la cual una de las estudiantes, se encuentra ante una dificultad sentida, la de comunicarse con un paciente sordomudo, situación problemática, ambigua, inesperada, que contiene elementos sorpresa, que implícitamente le propone una amplia gama de posibles alternativas de acción y que le exige tomar decisiones frente a sus diversas posibilidades (1).

“16:15h. Miriam entra a la habitación, tras sonar el timbre de llamada, es un hombre mayor de edad quien se lanzó de un segundo $\left(2^{\circ}\right)$ piso y fue arrollado por un coche sufriendo así fracturas de las costillas, por tanto su movilización es limitada, además es un paciente con déficit en la comunicación por ser sordomudo; sus familiares comentan que se agita y en ocasiones se desorienta en tiempo y espacio, lleva sujeción mecánica en las muñecas de sus brazos [...]. Este hombre quiere marcharse a casa, al parecer pregunta por sus pantalones, una de sus familiares gesticula lentamente con la boca y empleando el lenguaje de los signos, le dice que se encuentra en el hospital, y que 'Paco (otro familiar) se ha llevado el pantalón y las llaves a casa', se lo repite una y otra vez. La estudiante observa los modos de comunicación (gestos faciales y de la boca, movimientos de las manos) que emplea la familiar del paciente. Posteriormente, Miriam intenta comunicarse con él gesticulando lentamente palabras con la boca, explora distintas maneras de comunicarse con él, el paciente mueve insistentemente sus manos sujetas e 
intenta llevarlas hacia el abdomen; la estudiante al parecer no alcanza a comprender qué necesita el paciente" (Observación estudiante 4).

La estudiante se encuentra en una situación nueva y desconocida para ella, refiere sentimientos de impotencia y perplejidad ante la dificultad para comunicarse con el paciente. La estudiante hace una pausa en el interior de la situación para observar de manera deliberada el modo de comunicación que emplea la familia con el paciente, intenta conocer qué necesita el paciente, presta atención a la situación en sí, explora distintas maneras de comunicarse con él, hace inferencias al deducir lo que necesita el paciente a partir de la información que los familiares le suministran. Es decir, hace una suspensión de la incertidumbre, metafóricamente sería como el hecho de subirse a un árbol y desde allí poder obtener una visión más completa de la situación y decidir cómo lo hechos se relacionan entre sí (1). Es así que, hace un análisis y delibera situación, prestando atención a las necesidades del paciente, sacando a la superficie su comprensión intuitiva de los problemas de éste (la necesidad de comunicación, de eliminación y de movilización). Toma la decisión de acudir a la enfermera y allí le plantea sus inferencias (razonamientos) que permite entrever la deliberación que la estudiante ha realizado. La enfermera problematiza la situación del paciente y le da pistas respecto a las hipótesis o conjeturas a las que ha llegado la estudiante. La situación es compleja para ésta y existe un problema en la determinación real del problema del paciente (¿es un problema de eliminación, un episodio de desorientación o son ambas cosas?). Por otro lado, la estudiante mantiene una atención solícita y preocupación por dar respuesta a los familiares, como también por conocer ¿qué le pasa al paciente? y conectar con él. Ya que es a través del conocer y conectar con los pacientes que las enfermeras reconocen las distinciones cualitativas en las situaciones del paciente como base para la personalización del cuidado (16).

Mediante el diálogo y observación, la estudiante de enfermería aprende a conocer a los pacientes y obtiene de ellos la información necesaria acerca de sus necesidades, respuestas y sentimientos para tomar decisiones respecto a las acciones de cuidado requeridas. La relación de la estudiante con esta situación es transaccional, es decir, que ella tiene los pies dentro de la situación que está buscando comprender, da forma a la situación y conversa con ésta (3). Al indagar a la estudiante respecto a esta situación, afirma:

Me da impotencia porque tú sabes que él te quiere decir algo, pero claro, tampoco sabe leer, no sabe escribir, no puede comunicarse con nosotros y yo el lenguaje de signos, no lo sé (Entrevista estudiante 4).

La estudiante posteriormente se encuentra con el paciente y realiza un ejercicio de corroboración experimental (1), entabla una comunicación no-verbal con él, comprobando así las conjeturas e inferencias que ella ha elaborado. Esta conversación reflexiva que la estudiante mantiene en el interior de la situación es lo que se denomina "reflexión desde la acción" (3), una conversación que gira en espiral a través de etapas de apreciación, acción y reapreciación. 
[...] y luego por la noche otra vez me volvió a decir con gestos: 'que sus pantalones y sus llaves dónde estaban', y yo le decía: 'Paco se lo ha llevado a', claro yo le hacía los mismos gestos que la familiar y dijo: 'Ah vale, vale', [...] y yo me acuerdo que le dije: 'me lees los labios' y me dijo: 'sí', entonces yo le hablé pausadamente, [...] difícil porque es un paciente que no se puede expresar y comunicar contigo, es difícil, y yo estaba ahí con la impotencia de decir: 'me está diciendo algo y no sé qué es' [...] poco a poco tú desarrollas la capacidad de que te hagan un gesto y tú sepas qué es lo que quieren (Entrevista estudiante 4).

Obsérvese aquí, también esa predisposición de la estudiante a ser sensible a las necesidades del otro, evidenciado en el hecho de dar cuenta y atender a las necesidades más prioritarias para la persona en un espacio y tiempo determinado. Este aprendizaje experiencial le permite a la estudiante progresar hacia el desarrollo de una consciencia situada de las necesidades del Otro. Todas estas habilidades comunicativas como observar, escuchar, interpretar, hablar y expresar son requisitos ineludibles para la práctica de la enfermería, debido a que en muchos casos la comunicación enfermerapaciente se lleva a cabo bajo circunstancias que ofrecen barreras y distorsiones; pero estas mismas circunstancias llevan a que la comunicación no verbal adquiera un lugar preponderante (16).

\section{Curiosidad epistemológica}

El estudiante como sujeto partícipe del proceso de conocer en el que se encuentra se hace sujeto de la curiosidad. Una curiosidad necesaria para aprehender lo que se le está enseñando, que mueve al individuo a la creatividad, a expandir sus horizontes y a ser un sujeto vivo históricamente. Para Freire (17), esta curiosidad sin perder su estética se hace crítica, y ésta a su vez aproxima al individuo a la curiosidad epistemológica y metódica - a la investigación-.

Yo soy curiosa y lo reconozco, cuando llego donde mis pacientes yo me meto en la historia [...] me gusta mirar todo lo que tiene, por qué lo tiene, por qué viene, sus antecedentes, y digo: 'este señor tiene una traqueostomia ¿por qué le han hecho la traqueo?', y a lo mejor me pone 'parálisis diafragmática', y digo: 'eso es que no le va el diafragma y ¿por qué no le funciona?’, y empiezo a buscar, ¿por qué no le funciona el diafragma? (Entrevista estudiante 4).

Obsérvese la postura reflexiva-critica que asume la estudiante al indagar en las situaciones clínicas que se le presentan en el contexto de la práctica clínica, un contexto concreto y a la vez teórico (17). Una curiosidad que al ser metódica y rigurosa acerca al estudiante a los contenidos curriculares de la práctica clínica y a la experiencia de conocer (actividad gnoseológica) (17). 
El rol del profesor trasciende la lógica transmisora de conocimientos (discurso bancario), y se sitúa en un generador de preguntas y en un forjador de actitudes investigativas y de experimentación. Por tanto, el papel del educador es desafiar la curiosidad ingenua del educando para, con él, compartir la crítica, es así como la práctica educativa se afirma como reveladora de verdades ocultas. Y para ello, la práctica educativa ha de ser una experiencia dialógica $(17,18)$. Por otra parte, la curiosidad epistemológica de la estudiante que se evidencia en el anterior fragmento, es lo que se denomina un trabajo de detective (Detective Work) (16), central para el desarrollo del razonamiento clínico y la reflexión crítica de ésta. De igual manera, el seminario del prácticum es un contexto que viabiliza el espíritu de la curiosidad epistemológica del estudiante, y estimula el planteamiento de observaciones más focalizadas en su práctica clínica y a investigar su práctica:

\section{Ejemplo 1:}

Est. 7: Se podría estudiar hacer las curaciones de heridas de un grupo de paciente con yodo y el otro grupo sin yodo (Betadine ${ }^{\circledR}$ ).

Prof.: Sí, y a unos hacer la curación cada tanto de tiempo y a otros cada 6 horas, por ejemplo (Observación Seminario Estudiantes, Grupo I).

\section{Ejemplo 2:}

Est. 9: ¿Por la vía proximal va la Nutrición parenteral (NTP)?

Est. 8: La NTP va por la proximal, la Presión venosa Central (PVC) por la distal, en el Swan Ganz va por la distal [...]. La NTP tiene su luz propia, la proximal [Conflicto cognitivo respecto a la administración de la NTP].

Est. 9: Mañana lo pregunto y lo observo en la práctica. (Observación Seminario Estudiantes, Grupo I).

\section{Atención operativa}

El establecimiento de prioridades requiere un pensamiento "deliberado"' por parte de la estudiante, debido a que en un primer momento todas las tareas, peticiones y preocupaciones parecen estar en igual medida (16). Por tanto, averiguar cuáles son las tareas más urgentes resulta difícil debido a que aún no ha aprendido a ver el panorama general (the big picture) y no ha adquirido el sentido tácito de saliencia que hace que algunas cosas destaquen como más o menos importantes:

[...] Priorizar, siempre hacíamos lo que él [enfermero] iba diciendo, pues, me decia: 'hacemos esto, y luego esto', y claro, al dejarme la responsabilidad de priorizar, me lo tuve que pensar muy bien y luego a la hora de ir repartiendo, pues, pensar '¿qué haría el?', como lógica y, '¿qué puede ser lo mejor?', en ese caso lo mejor era retirar la sonda vesical porque 
así podíamos valorar la micción espontánea dentro de nuestro turno [...] (Entrevista estudiante 1).

La deliberación que la estudiante realiza en el momento de establecer prioridades deja entrever el estado de atención operativa que mantiene durante su práctica clínica; es decir, aquella disposición especial para trasladar lo que escucha a la acción, disposición que como se verá en los siguientes fragmentos, muestra la extraordinaria complejidad del proceso reflexivo de la estudiante: prestar atención a las descripciones y/o demostraciones de aspectos del cuidado que hace el tutor, para captar y retener lo que considera necesario aprender de estas actividades hacer una indagación y deliberación de las narraciones que ha escuchado, y tomar decisiones priorizando tal como ha escuchado y observado hacer al tutor. La estudiante hace un proceso de construcción selectiva de las cosas esenciales y no esenciales, que luego traslada a su propia ejecución (3).

\section{Entrevista Estudiante 1}

Habian pasado 3 dias antes, y él a mí me había explicado eso, entonces yo intento retener el máximo de información posible, luego busco entre ella, entre toda la información y decido qué es lo que me ha enseñado él que va primero (...) entonces pues eso, sólo recordar cuál es el momento para hacer cada cosa"

Inv.: ¿qué te facilita el que puedas retener lo que él te va diciendo?

Est. $1: j U y$ !, estar al cien por cien, con las orejas así, atentas - lleva sus dos manos detrás de sus orejas, y las mueve hacia adelante-y el cerebro trabajando todo el rato porque si te pierdes dos segundos ya has perdido el hilo de lo que te está explicando, entonces intentar captar, retener y preguntar, (...) pero intentar captar al máximo porque así luego todo se puede usar (Estudiante 1).

Es así que el "hacer" de la estudiante se presenta deliberado, razonado e inteligente (3). La estudiante hace una deliberación de las actividades a realizar, prioriza y organiza las actividades de cuidado y toma las decisiones de manera razonada.

\section{La Anticipación: conversación reflexiva en la acción}

La educación de enfermería se distingue por su énfasis en la práctica clínica, en saber qué, cómo y cuándo (16). Los educadores de enfermería hacen hincapié en la importancia de que los estudiantes entiendan las razones de sus acciones y en cómo ejecutar todos los pasos necesarios para la acción. 
Para ello, la estudiante hace tal como ha visto en el hacer de la tutora clinica. Esta habilidad de la estudiante para "hacer lo que ve hacer" a otra persona, le faculta para reproducir elementos de una actividad cuyo significado aún no entiende (3). Cuando ejecuta estas actividades, las experimenta sintiendo cómo son y descubriendo en ellas, mediante la reflexión, significados que no había sospechado anteriormente:

Pues, el primer pensamiento es organización, primero pienso 'a ver Sonia por pasos, ¿qué materiales necesitas?', y a la hora de hacer el procedimiento me acuerdo de la persona que me enseño aquello, si fue una buena enseñanza, porque la gasometría arterial siempre me acuerdo de Joan (enfermero) [...] diciéndome: 'coge el material, prepáralo tal', entonces intento en cada cosa que hago pensar '¿qué me enseñaron, qué fue lo que me enseñaron?' (Entrevista estudiante 2).

Puede apreciarse aquí que la estudiante adopta una disposición mental que le permite prestar atención a las descripciones y/o demostraciones del tutor clínico y, al ejecutar el procedimiento aprende de manera significativa. Este aprender haciendo extiende el alcance imaginativo de la estudiante; es decir, el desarrollo de su capacidad para evaluar a sus pacientes como se presentan físicamente y anticiparse al próximo pasó en su cuidado (16). Lo que favorece la interiorización y desarrollo de su conocimiento práctico, de modo tal que retoma las descripciones y/o demostraciones del tutor que ha visto y oído y reconstruye la acción en posteriores ejecuciones seleccionando, repasando la información que ha considerado esencial de la enseñanza del tutor:

[...] cuando estoy con él (enfermero) es como que me confío un poco más, en cambio, cuando estoy sola es como todo el rato pensando, ¿qué tengo que hacer?, siguiente paso y es un ejercicio, bueno, para asimilarlo todo. [...] repaso en mi memoria lo que he utilizado las veces anteriores, [...] y voy haciendo. Lo que hago mucho es como tener conversaciones conmigo misma, lo que hablaría con él (enfermero) [...] así no me salto ningún paso (Entrevista estudiante 1).

Tal como la estudiante expresa, lo que ella hace en el interior de la acción es tener conversaciones reflexivas consigo misma, repasa en su memoria las experiencias anteriores, los materiales que ha empleado en éstas, los pasos o diversas posibilidades de acción que ha realizado u observado de ella o de su tutor; reflexiona y asigna significado a su acción y se anticipa de manera reflexiva en su hacer; es así que, mantiene un estado de atención operativa que le permite trasladar a la acción, haciendo aquello que ella ha visto u oído en su práctica clínica. En definitiva, la atención operativa que la estudiante mantiene en el interior de la acción, le lleva a hacer una conversación reflexiva en la acción donde enlaza las experiencias previas, la situación presente y se anticipa al paso siguiente: 
Siempre la memoria de las veces anteriores, siempre pienso: 'a ver, ¿qué he visto?, ¿cómo lo ha hecho el enfermero?', y también me anticipo a los pasos, digo: 'vale, si el siguiente paso se hace esto, necesito eso', entonces voy a coger eso y 'sí para hacer esto necesitare esto, por ejemplo, para conectar eso, pues tengo que tener las pinzas a mano' y ya me voy adelantando (Entrevista estudiante 1).

A medida que repite no sólo las acciones constitutivas, sino también las reflexiones en la acción, puede que la estudiante descubra en algún punto que ha interiorizado la ejecución (3). Uno de los rasgos centrales de una práctica inteligente lo constituye la capacidad de anticipación, es decir, la capacidad para salir airoso de situaciones imprevistas (2).

\section{Imitación reflexiva}

Cuando en una práctica de tutoría se coordina el demostrar y el imitar, el decir y el escuchar, cada proceso constitutivo llena vacíos de significados inherentes al otro (3). Las demostraciones y descripciones, las comparaciones del proceso y del producto, proporcionan el material para la mutua reflexión en la acción. En la siguiente observación la estudiante adopta una actitud de atención operativa delante del decir y de las demostraciones que el enfermero le proporciona; capta lo que ella cree que necesita aprender de las demostraciones (y del decir) que hace el tutor, adquiriendo así capacidad para imitar:

La estudiante verifica que el nombre del paciente coincida con el nombre que aparece en la bolsa de NTP, luego de que el enfermero J. le recordara esta precaución 'siempre tú verifica que es el paciente'. Seguidamente la estudiante sale de la habitación para el lavado estéril de sus manos, el enfermero antes de salir le acerca un paquete de gasas, necesario para el secado de sus manos. Al cabo de un momento la estudiante ingresa en la habitación tras el lavado de sus manos y se coloca los guantes estériles. El enfermero pone la bomba de infusión de la NTP en pausa, indicándole que también puede pausar ella la bomba estando con los guantes estériles con una gasa estéril (Le da otra posibilidad de acción).

La estudiante realiza el procedimiento siguiendo los pasos que ha observado del enfermero en anteriores cambios de bolsa de NTP (Observación estudiante 1).

Seguidamente el enfermero permite que la estudiante realice el procedimiento de manera autónoma, una vez que le ha proporcionado los elementos fundamentales del procedimiento y ha observado a la estudiante realizar el procedimiento:

"Enfermero: 'bueno, el otro cambio de bolsa de NTP la vas a hacer tú, yo me voy y la haces tú sola ¿Qué te parece? [...]”' (Observación estudiante 1). 
Para Schön (3), imitar es, en cierto modo, experimentar, ya que, si quiere "seguir", el estudiante debe construir en su propia ejecución aquello que él ejecuta como rasgos esenciales de la demostración del tutor. Por tanto, la imitación reflexiva adquiere un carácter exploratorio:

El enfermero J. se retira de la habitación. Irene comienza con la preparación del material para el otro cambio de bolsa de NTP. La observo segura al momento de preparar el material, aprecio su mirada atenta y emotiva por la situación, pensando y preguntándose por lo que está haciendo, en voz alta me explica sobre el material que prepara, la realización del procedimiento y también el planteamiento de preguntas que se hace a sí misma sobre el próximo paso que ha de tener en cuenta (Observación estudiante 1).

Por tanto, la reconstrucción por imitación de una acción observada es un tipo de solución de problemas que puede tomar la forma de diferenciaciones sucesivas de un procedimiento global (es decir, las semejanzas y diferencias entre el procedimiento original y la copia que la estudiante hace de ello) o de aprender a engarzar acciones o pasos constituyentes del procedimiento (3). El proceso de construcción es, no obstante, una forma de reflexión en la acción, una indagación sobre la marcha en la que la estudiante construye y comprueba en su propia acción, hasta detectar que ésta se ajusta al proceso que ha observado. En este punto, puede intentar hacerlo de nuevo, dirigiendo ahora su esfuerzo de imitación a su propia acción recién finalizada. Progresa desde la imitación del otro a la imitación de sí mismo (3).

La estudiante ahora está en condiciones de reflexionar acerca de su propio proceso, preguntando qué reglas, operaciones y conocimientos ha desarrollado y comparándolos con las primeras descripciones del enfermero (3). Lo que empezó como una reconstrucción por imitación de la acción del enfermero, lo experimenta ahora como algo propio de ella, un elemento nuevo de su propio repertorio disponible para su utilización, por medio del ver-como y el hacer-como. Es a través de la imitación reflexiva, que la estudiante trata de introducir en la ejecución de sus propias acciones aquellos rasgos característicos que ha visto en la demostración de su tutor; improvisa, también, sobre la marcha, experimentos espontáneos que le sirven para descubrir y verificar lo que el tutor puede estar tratando de comunicarle. "Y, para hacer todo esto, el alumno adopta un determinado tipo de actitud; la de responsabilizarse por formarse a sí mismo en aquello que necesita aprender y, al mismo tiempo, mantenerse dispuesto a recibir la ayuda del tutor" (4).

\section{Conclusiones}

La complejidad del prácticum clínico favorece el desarrollo y la integración de procesos reflexivos en el estudiante de enfermería, como: la capacidad perceptiva y consciencia situada de las necesidades del otro; la curiosidad epistemológica; la atención operativa; la anticipación: conversación reflexiva 
en la acción; y la imitación reflexiva. Estos procesos reflexivos se manifiestan en el estudiante cuando: se desenvuelve reflexivamente delante de las situaciones inciertas, complejas de la práctica, y de los cambios que pueden tomar cada situación clínica; plantea las situaciones como un caso único; se responsabiliza del cuidado de la otra persona; mantiene conversaciones reflexivas y críticas con los materiales de la situación; atiende de manera operativa y descifra los significados, interpretaciones y rasgos esenciales de la descripciones y demostraciones de la enfermera; toma decisiones deliberadas y razonadas, las cuales son a su vez éticas y estéticas; centra la atención en su acción, improvisando y explorando en su propia ejecución, a través de la imitación reflexiva, la cual adquiere un carácter explorador y reflexivo en la acción del estudiante. De modo que éste progresa desde la imitación del enfermero a la imitación de sí mismo.

\section{Financiamiento}

COLCIENCIAS: LASPAU, Convocatoria N419, expediente $\mathrm{n}^{\circ} 20080404$.

\section{Conflicto de intereses}

Ninguno

\section{Agradecimientos}

A la Universidad Nacional de Colombia, a Colciencias, a la Universidad de Barcelona y al Hospital Universitario de Bellvitge por el apoyo brindado para la realización del presente trabajo.

\section{Referencias}

1. Dewey J. Cómo pensamos. Nueva exposición de la relación entre pensamiento reflexivo y proceso educativo. Barcelona: Paidós Ibérica S.A.; 2007.

2. Bárcena F, Larrosa J, Mélich JC. Pensar la educación desde la experiencia. Revista portuguesa de pedagogía. 2006; 40 (1): 233-259.

3. Schön D. La formación de profesionales reflexivos. Hacia un nuevo diseño de la formación y el aprendizaje en las profesiones. Madrid: Paidós Ibérica S.A; 1992.

4. González CX. Formación del pensamiento reflexivo en estudiantes universitarios. Magis. Revista Internacional de Investigación en Educación. 4 (9): 595-617. [Internet] 2012 [citado 20 diciembre 2015]. Disponible en: http://www.redalyc.org/articulo.oa?id=281022848005

5. Bardallo M. Entre la enseñanza y el aprendizaje: Un espacio de saberes para compartir. La tutoría en Enfermería [tesis doctoral]. Barcelona: Universitat de Barcelona / Programa de Pedagogía; 2010.

6. Queirós PJP. The knowledge of expert nurses and the practical-reflective rationality. Invest Educ Enferm. 2015; 33 (1): 83-91.

7. Rodríguez M. El arte del cuidado: Un legado reflexivo de saber [tesis doctoral]. Madrid: Universidad Europea de Madrid / Programa de Enfermería; 2012. 
8. Scanlan JM, Care WD, Udod S. Unravelling the unknowns of reflection in classroom teaching. Journal of Advanced Nursing. 2002; 38 (2): 136-143.

9. Solvoll B, Heggen KM. Teaching and learning care. Exploring nursing students' clinical practice. Nurse Educ Today. 2010; 30 (1): 73-77.

10. Tseng $H$, Wang $H$, Weng W. Nursing students' perceptions toward the nursing profession from clinical practicum in a baccalaureate nursing program. A qualitative study. The Kaohsiung Journal of Medical Sciences. 2013; 29 (3): 161-168. doi:10.1016/j.kjms.2012.08.027

11. Wilson A. Mentoring student nurses and the educational use of self: A hermeneutic phenomenological study. Nurse Educ Today. 2014; 34 (3): 313-318.

12. Van Manen M. Investigación educativa y experiencia vivida: Ciencia humana para una pedagogía de la acción y la sensibilidad. Barcelona: Idea Books; 2003.

13. Strauss AL, Corbin J. Bases de la investigación cualitativa: Técnicas y procedimientos para desarrollar la teoría fundamentada. Medellín: Editorial Universidad de Antioquia; 2002.

14. Declaración de Helsinki de la Asociación Médica Mundial. Principios éticos para las investigaciones médicas que involucran seres humanos. 59a Asamblea General. Seúl, Corea. [Internet] 2008 [citado 15 octubre 2010]. Disponible en: http://www.ub.edu/recerca/Bioetica/ doc/Declaracio_Helsinki_2008.pdf

15. Ley de Orgánica 15/1999, de Protección de Datos de Carácter Personal (LOPD). (BOE núm. 298 \& 23750, 13 de diciembre de 1999).

16. Benner P, Sutphen M, Leonard V, Day L. Educating nurses. A call for radical transformation. 1a ed. United States of America: JosseyBass; 2010.

17. Freire P. Cartas a quien pretende enseñar. 11a. ed. México: Siglo XXI Editores S.A.; 2006

18. Waterkemper R, do Prado ML, Medina JL, Schmidt, K. Development of critical attitude in fundamentals of professional care discipline: A case study. Nurse Educ Today. 2014; 34 (4): 581 - 585. 